\title{
Evidence for gamma-ray jets in the Milky Way
}

\section{Citation}

Su, Meng, and Douglas P. Finkbeiner. 2012. "Evidence for gamma-ray jets in the Milky Way." The Astrophysical Journal 753 (1) (June 14): 61. doi:10.1088/0004-637x/753/1/61.

\section{Published Version}

10.1088/0004-637x/753/1/61

\section{Permanent link}

http://nrs.harvard.edu/urn-3:HUL.InstRepos:34519625

\section{Terms of Use}

This article was downloaded from Harvard University's DASH repository, and is made available under the terms and conditions applicable to Other Posted Material, as set forth at http:// nrs.harvard.edu/urn-3:HUL.InstRepos:dash.current.terms-of-use\#LAA

\section{Share Your Story}

The Harvard community has made this article openly available.

Please share how this access benefits you. Submit a story.

\section{Accessibility}




\title{
EVIDENCE FOR GAMMA-RAY JETS IN THE MILKY WAY
}

\author{
Meng Su ${ }^{1}$ and Douglas P. Finkbeiner ${ }^{1,2}$ \\ ${ }^{1}$ Institute for Theory and Computation, Harvard-Smithsonian Center for Astrophysics, 60 Garden Street, MS-51, \\ Cambridge, MA 02138, USA; mengsu@cfa.harvard.edu \\ 2 Physics Department, Harvard University, Cambridge, MA 02138, USA \\ Received 2012 February 9; accepted 2012 April 5; published 2012 June 14
}

\begin{abstract}
Although accretion onto supermassive black holes in other galaxies is seen to produce powerful jets in X-ray and radio, no convincing detection has ever been made of a kpc-scale jet in the Milky Way. The recently discovered pair of $10 \mathrm{kpc}$ tall gamma-ray bubbles in our Galaxy may be signs of earlier jet activity from the central black hole. In this paper, we identify a gamma-ray cocoon feature in the southern bubble, a jet-like feature along the cocoon's axis of symmetry, and another directly opposite the Galactic center in the north. Both the cocoon and jet-like feature have a hard spectrum with spectral index $\sim-2$ from 1 to $100 \mathrm{GeV}$, with a cocoon total luminosity of $(5.5 \pm 0.45) \times 10^{35}$ and luminosity of the jet-like feature of $(1.8 \pm 0.35) \times 10^{35} \mathrm{erg} \mathrm{s}^{-1}$ at $1-100 \mathrm{GeV}$. If confirmed, these jets are the first resolved gamma-ray jets ever seen.
\end{abstract}

Key words: galaxies: active - galaxies: starburst - gamma rays: galaxies - ISM: jets and outflows

Online-only material: color figures

\section{INTRODUCTION}

Supermassive black holes (SMBHs) of $10^{6}-10^{10}$ solar masses are believed to lie at the center of most galaxies and are fed by accretion of ambient gas and stars. Accretion-powered jets have been observed at various astronomical scales ranging from active galactic nuclei (AGNs; Bridle \& Perley 1984), especially blazars (BL Lac objects and flat-spectrum radio quasars) at the bright end, to gamma-ray bursts and Galactic binaries (stellar mass black holes, neutron stars, and cataclysmic variables). Some of these objects appear to have produced jets nearly continuously for at least tens of millions of years. The mechanism by which jets turn on and off is one of the major puzzles in high-energy astrophysics, and may be connected to star formation (Antonuccio-Delogu \& Silk 2008). The relativistic jets inject significant amounts of energy into the medium within which they propagate, creating an extended, underdense, and hot cocoon. After decades of study, we still lack a complete understanding of the main mechanism launching, accelerating, and collimating jets, with limited knowledge of the energy content, the composition, and the particle acceleration mechanisms of the jets (Blandford \& Znajek 1977; Blandford \& Payne 1982).

The SMBH at the center of the Milky Way (MW) is surrounded by clusters of young stars and giant molecular clouds (Morris \& Serabyn 1996). Although there are indications of past activity (Sunyaev et al. 1993), the SMBH is currently in a quiescent state. Despite the abundant observational evidence of large-scale jets in other galaxies, it was not expected that the MW's SMBH would produce such a relativistic collimated structure, given its current quiescence. However, the MW must have undergone phases of nuclear activity in the past in order for the SMBH to grow, and it is plausible that signs of past activity are still visible. One might expect relics of past activity in high-energy cosmic rays (CRs) and hot gas, perhaps far off the disk. The sensitivity and angular resolution of the Large Area Telescope (LAT) on board the Fermi Gamma-ray Space Telescope (Atwood et al. 2009) make possible the search for inverse Compton (IC) gamma rays from a Galactic jet.
In this work, we use LAT data at $0.3 \mathrm{GeV}<E_{\gamma}<100 \mathrm{GeV}$ to look for unexpected diffuse Galactic gamma-ray structure. We will show evidence for a large-scale-collimated double-jet structure, which appears symmetric with respect to the Galactic center (GC). For the remainder of this paper, we refer to these collimated jet-like structures as the "gamma-ray jets" or simply the "jets." We also argue that the southern jet has produced a large cocoon feature, visible over a wide range of gamma-ray energies. These jets and cocoon features might be associated with the previously discovered Fermi bubble structures (Su et al. 2010), and may hold the key to understanding their origin.

In Section 2, we describe the Fermi-LAT data selection and our data analysis procedure including map making. In Section 3, we describe our model of the Galactic diffuse gamma-ray emission. In Section 4, we show that the gammaray maps constructed from three-year Fermi-LAT observations reveal evidence of large-scale Galactic jet features along with a south cocoon structure. We characterize the morphology of the jet/cocoon system in some detail and employ regression template fitting to determine the jet and cocoon spectra in Section 5. We calculate and discuss the radio luminosity of the jet in Section 6 and carefully discuss the statistical significance of the jet structure in Section 7. Finally, we discuss the implications of the presence of the Galactic jet and future observations in Section 8 .

\section{MAP CONSTRUCTION FROM FERMI-LAT DATA}

\subsection{Fermi Data Selection}

The LAT (Gehrels \& Michelson 1999; Atwood et al. 2009) is the principal scientific instrument on board the Fermi Gammaray Space Telescope. The point-spread function (PSF) is about 0.8 for $68 \%$ containment at $1 \mathrm{GeV}$ and decreases with energy as $r_{68} \sim E^{-0.8}$, asymptoting to $\sim 0.2$ at high energy. It is designed to survey the gamma-ray sky in the energy range from about $20 \mathrm{MeV}$ to several hundreds of $\mathrm{GeV}$.

Using standard photon event selection (zenith angle $<100^{\circ}$, etc.), we generate full-sky maps of counts and exposure using HEALPix, a convenient equal-area iso-latitude full-sky 

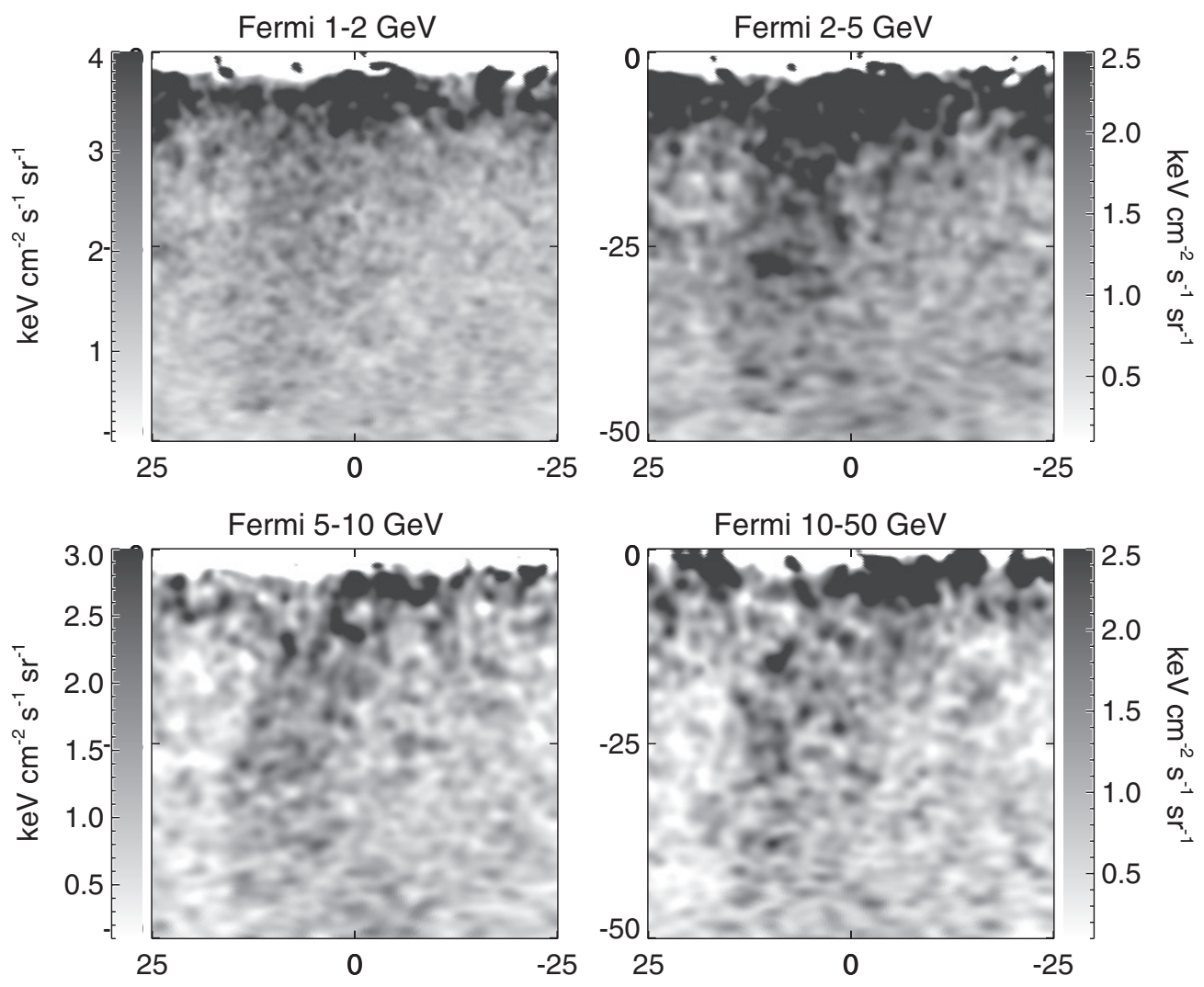

Figure 1. Large-scale gamma-ray cocoon feature is revealed by template decomposition of the gamma-ray maps in energy range of $1-2 \mathrm{GeV}$ (upper left), $2-5 \mathrm{GeV}$ (upper right), 5-10 GeV (lower left), and 10-50 GeV (lower right) maps constructed from Fermi-LAT three-year observations. Bright point sources have been masked and fainter ones subtracted. The maps are smoothed by a Gaussian kernel with FWHM of 90 arcmin. We remove the dominant diffuse Galactic signal from $\pi^{0}$ (and bremsstrahlung) gamma rays produced by CR protons (electrons) interacting with the ISM using a dust map template constructed based on far-IR data (Schlegel et al. 1998). A smooth disk model is also subtracted to reveal the structure deeper into the plane. This model mostly removes the IC gamma rays produced by CR electrons interacting with the interstellar radiation field including CMB, infrared, and optical photons. We also subtract a uniform template of the Fermi bubbles to remove the corresponding gamma-ray emission. The cocoon feature is revealed on the east (left) side of the previously discovered southern Fermi bubble structure (Su et al. 2010), with relatively sharp edges.

pixelization widely used in the cosmic microwave background (CMB) community. ${ }^{3}$ Spherical harmonic smoothing is straightforward in this pixelization, and we smooth each map by the appropriate kernel to obtain a Gaussian PSF of $2^{\circ}$ FWHM. Because the PSF of the initial map must be smaller than this, at energies from $300 \mathrm{MeV}$ to $1 \mathrm{GeV}$ we use only front-converting events, which have a smaller PSF. Above $1 \mathrm{GeV}$, we combine front- and back-converting events.

We make use of the Fermi Pass 7 (P7) data products, using the latest publicly available event reconstruction algorithms. ${ }^{4}$ For the purpose of studying Galactic diffuse gamma-ray emission with minimal non-photon contamination (low background), we select events designated ULTRACLEAN ${ }^{5}$ class that have the most stringent data selection criteria, so that any diffuse features appearing on the gamma-ray maps are not due to CR contamination. Photons coming from the bright limb at Earth's horizon, dominantly produced by grazing-incidence CR showers coming directly toward the LAT, are a strong source of contamination. We minimize this background by restricting

\footnotetext{
3 HEALPix software and documentation can be found at http://healpix.jpl. nasa.gov, and the IDL routines used in this analysis are available as part of the IDLUTILS product at http://sdss3data.lbl.gov/software/idlutils.

4 Details at http://fermi.gsfc.nasa.gov/ssc/.

5 Maps produced with CLEAN have about $20 \%$ more events. Repeating our analysis on these maps yields similar results, with similar error bars at

$E \lesssim 10 \mathrm{GeV}$, and slightly larger error bars at $E \gtrsim 10 \mathrm{GeV}$, where background is relatively more important.
}

to events with zenith angle less than $100^{\circ}$ as suggested in the Fermi Cicerone. ${ }^{6}$

\subsection{Map Making}

Our current gamma-ray maps (v3_3) constructed from the three-year Fermi data have greater signal/noise and significantly lower background compared to the previously released v2_3 maps in Su et al. (2010). The "three-year maps" exclude some short time periods, primarily while Fermi passes through the South Atlantic Anomaly. We construct maps using the newly released photon event list corresponding to the P7_V6 Instrument Response Functions (IRFs).

As in Su et al. (2010), we construct maps of front-converting and back-converting events separately, smooth to a common PSF, and then combine them. To reveal the diffuse emission, we subtract point sources using the Second Fermi-LAT catalog (2FGL), which is based on 24 months of LAT observations ${ }^{7}$ and the P7_V6 event selections and IRFs. The PSF and effective area of the Fermi-LAT vary with energy, and we subtract each point source from the maps in each energy bin, using the in-flight version of the PSF contained in the P7_V6 IRFs. We produce the exposure maps using the gtexpcube task in the Fermi Science Tools.

\footnotetext{
6 http://fermi.gsfc.nasa.gov/ssc/data/analysis/documentation/

7 http://fermi.gsfc.nasa.gov/ssc/data/access/lat/2yr_catalog, the file we used is gll_psc_v05.fit.
} 

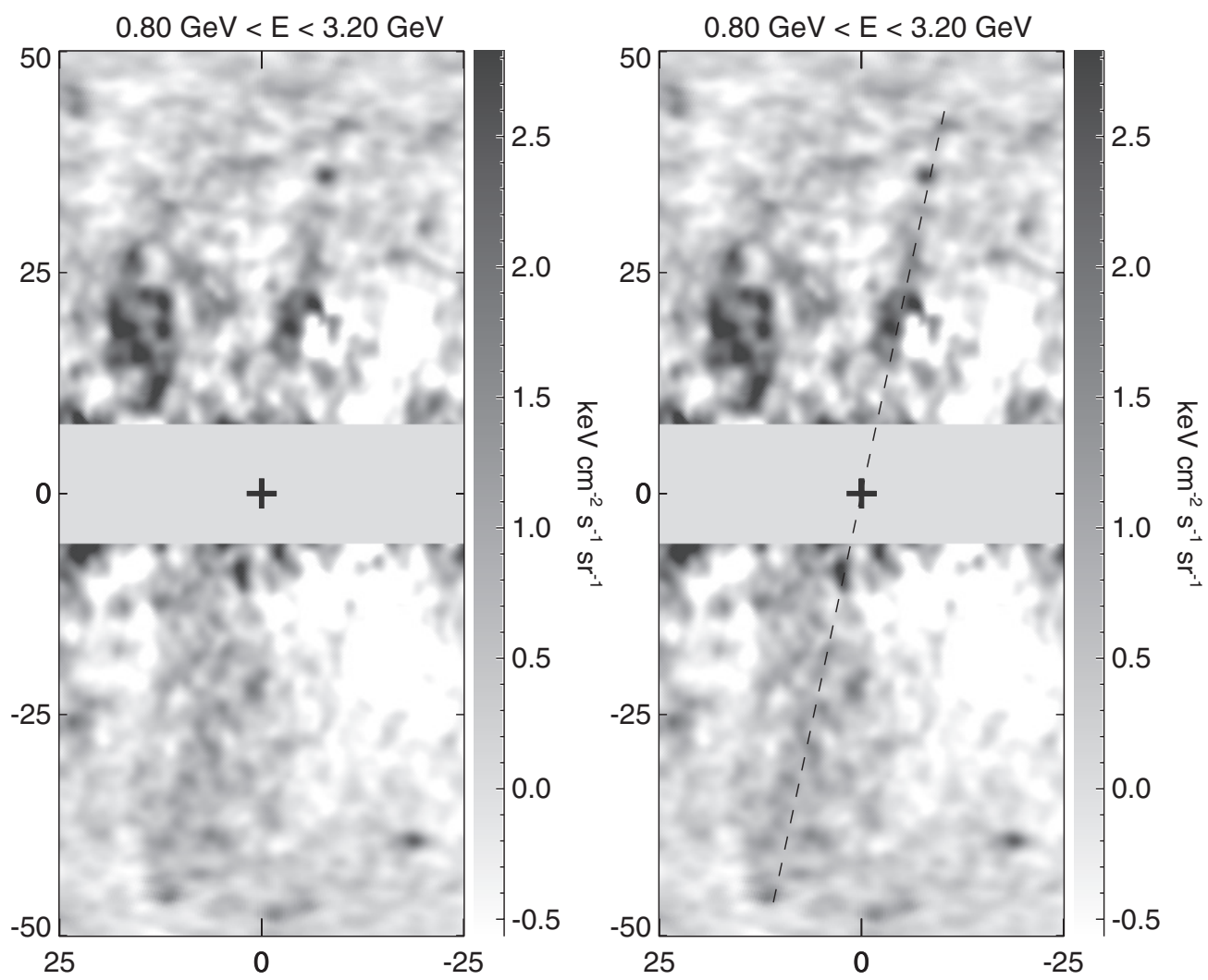

Figure 2. We show gamma-ray maps in Galactic coordinate $\left(-50^{\circ}<b<50^{\circ},-25^{\circ}<\ell<25^{\circ}\right)$ from Fermi-LAT for photon energies $0.8-3.2 \mathrm{GeV}$. The Galactic center is marked with a cross sign in the center of the maps. Two large-scale-collimated jet-like features (the gamma-ray jets) are revealed. The right panel shows the same image as the left panel, but with a dashed line representing the direction of the suspected jet. Point sources have been subtracted based on the Second Fermi-LAT catalog (2FGL; The Fermi-LAT Collaboration 2011), and large sources, including the inner disk $\left(-5^{\circ}<b<7^{\circ}\right)$, have been masked. The maps are smoothed by a Gaussian kernel with FWHM of $2^{\circ}$. The Fermi diffuse Galactic model (Pass7_V6) has been subtracted to remove known large-scale diffuse gamma-ray emission.
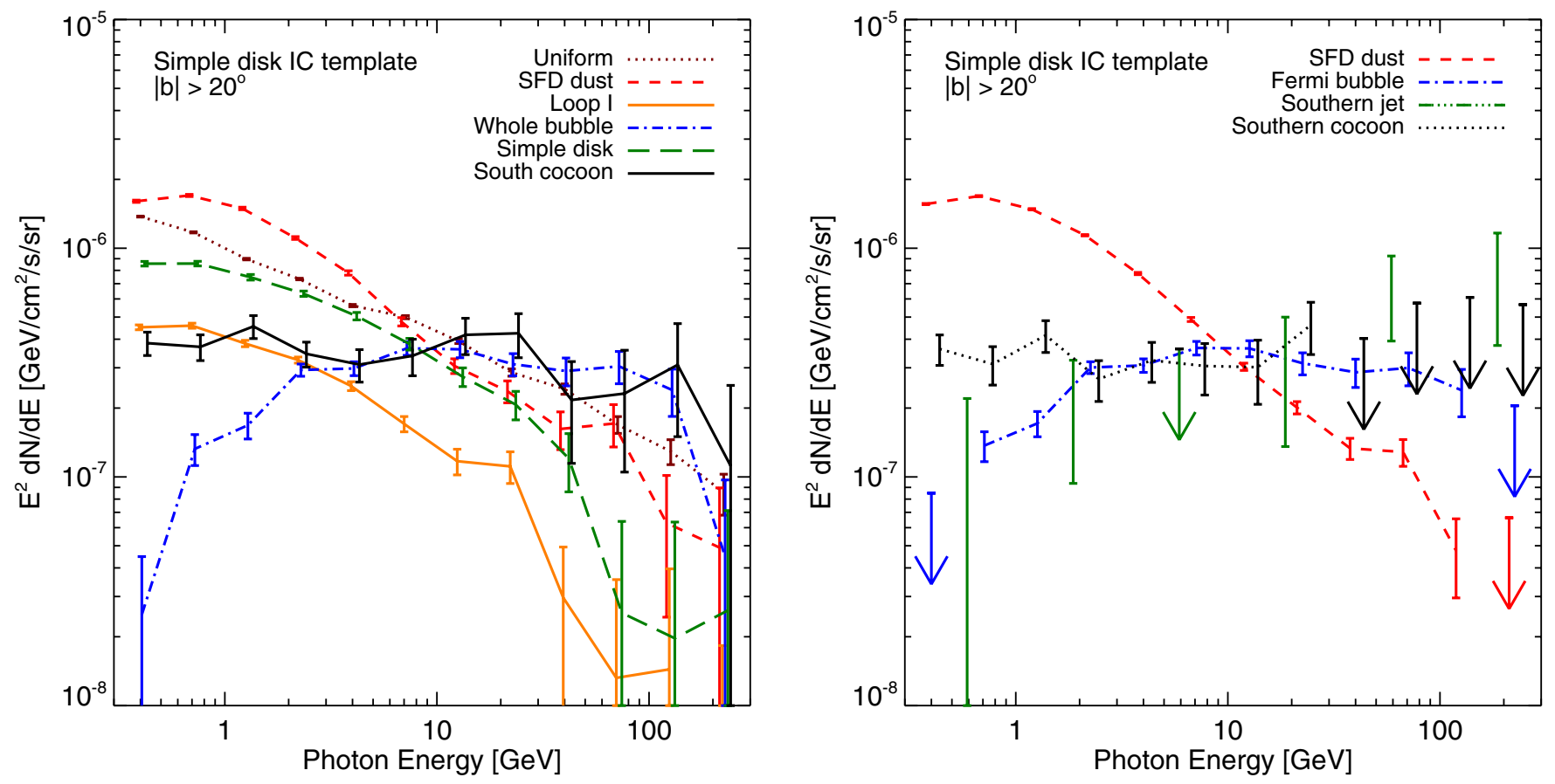

Figure 3. Left panel: spectral energy distribution of the components in our six-template fit. The dust-correlated spectrum (red short dashed line) traces $\pi^{0}$ emission. The disk-correlated emission (green dashed) approximately traces the soft inverse Compton and bremsstrahlung components. The spectrum of the uniform emission (dotted brown line) includes the isotropic part of the extragalactic background and CR contamination. The spectrum of emission correlated with Loop I (solid orange) has a spectrum similar to the disk-correlated emission. In contrast to these soft-spectrum components, the Fermi bubble template (blue dot-dashed) and the gamma-ray cocoon (black solid) have notably harder (consistent with flat) SEDs. Vertical bars show the marginalized 68\% confidence range derived from the parameter covariance matrix for the template coefficients in each energy bin. Right panel: same, but with one additional template representing the gamma-ray jet. For clarity, we only show the spectrum of the gamma-ray jet, the cocoon, and the Fermi bubbles, and compare their spectra with the softer $\pi^{0}$ emission (red dashed).

(A color version of this figure is available in the online journal.) 

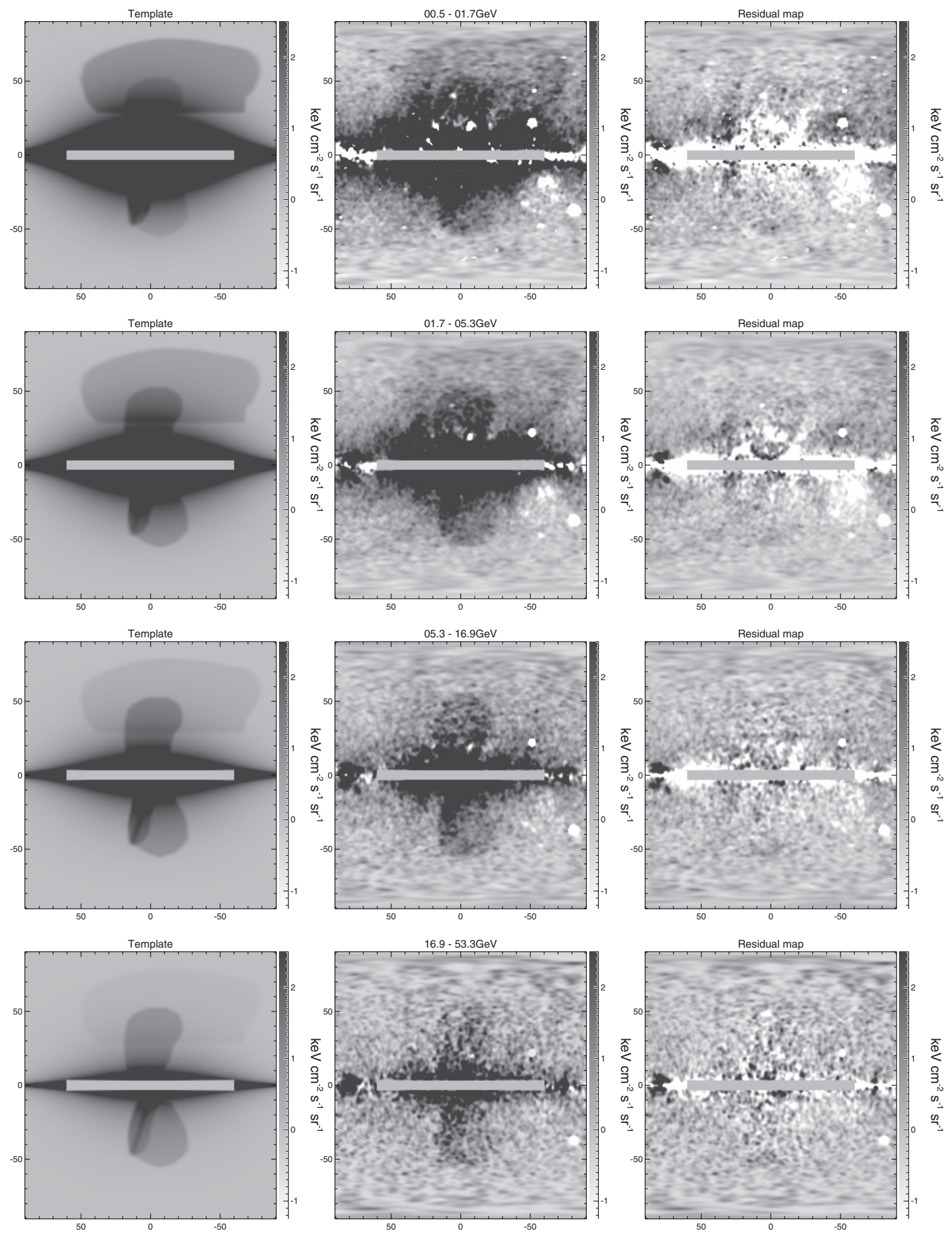

Figure 4. This figure shows the best-fit linear combination maps corresponding to the spectra in Figure 1. To increase signal/noise, larger energy bins are used. The left column shows the linear combination of the disk, Loop I, uniform, bubble template, the south cocoon, and the south jet template, that provide the best fit to the Fermi maps (middle column) after subtracting the best-fit SFD dust template. Because the $\pi^{0}$ emission traced by SFD is so bright, it is subtracted from both the models and data shown in this figure. The difference maps (data minus template model) are in the right column. The template fitting is done for the region with $|b|>20^{\circ}$ to avoid contamination from the Galactic disk. The subtraction of the model largely removes the features seen in the Fermi maps with $|b|>20^{\circ}$. We have also masked the inner Galactic plane region $\left(|b|>4^{\circ}\right.$ and $\left.|l|>60^{\circ}\right)$, which is significantly contaminated by point sources. We use the same gray scale for all panels. We find that both the disk IC template and Loop I features fade away with increasing energy, but the jet template does not. The oversubtraction in the residual maps, especially in the lower energy bins, is due to the simple disk IC model, which is not a good template across the entire disk. However, in the fit region $\left(|b|>20^{\circ}\right)$, the residual maps are consistent with Poisson noise without obvious large-scale features. 


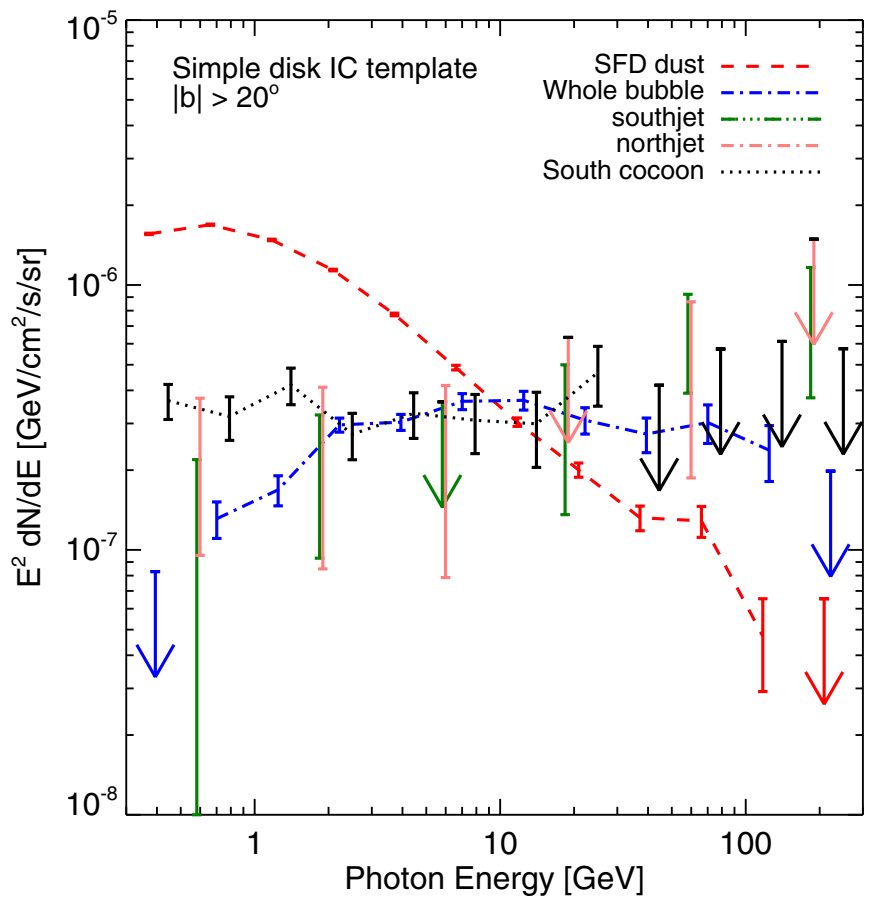

Figure 5. This figure is the same as Figure 3, except with the north jet template added to the regression. The north and south jet spectra are noisy, but consistent with each other, and consistently hard. Note that the upper limit is shown for $3 \sigma$. The spectra plotted here are available in Tables 1 and 2 .

(A color version of this figure is available in the online journal.)

In principle, the PSF of each event depends on the angle between its arrival direction and the instrument axis. The LAT scan strategy results in a small variation of the PSF with position on the sky. We use the in-flight determination of the PSF that neglects these details. ${ }^{8}$ For the 400 brightest and 400 most variable sources, the subtraction is noticeably imperfect, so we interpolate over the core of the PSF after subtracting the best estimate. We take care to expand the mask for very bright sources (Geminga, 3C 454.3, and LAT PSR J1836+5925) and large sources (Orion and the Magellanic Clouds). The resulting map is appropriate for the analysis of diffuse emission at $|b|>3^{\circ}$. At $|b|<3^{\circ}$, the maps are severely compromised by the poor subtraction and interpolation over a large number of point sources. Further details of the map processing may be found in Dobler et al. (2010) and Su et al. (2010). The v3_3 maps used in this work are available for download ${ }^{9}$ in both FITS and jpeg formats.

\section{DIFFUSE GALACTIC GAMMA-RAY EMISSION MODELS}

At low $(\sim 1 \mathrm{GeV})$ energies, and near the Galactic plane $\left(|b|<20^{\circ}\right)$, the gamma rays observed by Fermi-LAT are dominated by photons from the decay of $\pi^{0}$ particles, produced by the collisions of CR protons with ambient gas and dust in the interstellar medium (ISM). Collisions of CR electrons with the ISM (primarily protons, but also heavier nuclei) produce bremsstrahlung radiation. The $\mathrm{CR}$ electrons also IC scatter the interstellar radiation field (ISRF) up to gamma-ray energies. In order to reveal the gamma-ray jet features, significant $\pi^{0}$ emission, bremsstrahlung, and IC emission from the Galactic disk

\footnotetext{
8 See http://fermi.gsfc.nasa.gov/ssc/data/analysis/documentation/Cicerone/ Cicerone_LAT_IRFs/IRF_PSF.html.

9 Available at http://fermi.skymaps.info.
}

must be removed. We take two approaches to this foreground removal: one is to use the Fermi Diffuse Galactic Model ${ }^{10}$ provided by the Fermi team; the second is to employ a linear combination of templates of known emission mechanisms, using existing maps from multi-wavelength observations and/or constructed geometric templates.

\subsection{Fermi Diffuse Galactic Model}

The LAT Diffuse Galactic Model is a comprehensive model of Galactic gamma-ray emission from the ISM. ${ }^{11}$ The Fermi diffuse model is primarily designed as a background template for point-source analysis or investigation of small-scale diffuse structures, and comes with a number of caveats. However, these caveats apply mainly near the Galactic plane, and at $E>50 \mathrm{GeV}$. It is nevertheless useful for qualitatively revealing features in the diffuse emission at high latitude. In this work, we use the version of Fermi diffuse Galactic model gal_2yearp7v6_v0.fits.

\subsection{Simple Template-based Diffuse Galactic Model}

The $\pi^{0}$ /bremsstrahlung gamma-ray intensity is proportional to the ISM density $\times$ the CR proton/electron density, integrated along the line of sight. As long as the CR proton/electron spectrum and density are approximately spatially uniform, the ISM column density is a good tracer of the resulting gammaray distribution from $\pi^{0} /$ bremsstrahlung emission. Because the emission is optically thin, subtracting a simple template of the $\pi^{0}$ gammas helps to reveal the gamma-ray jets/cocoon along with the Fermi bubbles, especially toward the Galactic plane. The ISM column density is expected to be strongly correlated with other tracers of the ISM. Our previous work ( $\mathrm{Su}$ et al. 2010) used the Schlegel, Finkbeiner, \& Davis (SFD; 1998) map of Galactic dust, based on far-IR data (Schlegel et al. 1998). The dust map has some advantages over gas maps. One shortcoming of using H I and CO maps is the existence of "dark gas" (Grenier et al. 2005), clouds with gamma-ray emission that do not appear in the H I and CO surveys. These features are seen in dust maps (Schlegel et al. 1998) and may simply be molecular H clouds underabundant in CO. For the dust map, there are no problems with self-absorption, thus no concerns about "dark gas," and the SFD dust map has sufficient spatial resolution (SFD has an FWHM of $6^{\prime}$, and LAB H I is $36^{\prime}$ ). On the other hand, SFD contains no velocity information, so it is impossible to break the map into Galactocentric rings as is done with $\mathrm{H}$ I and CO maps. Nevertheless, it is instructive to employ the SFD map to build a very simple Galactic diffuse gamma-ray template. The goal is to reveal the gamma-ray jet/cocoon structures by removing the expected diffuse emission in a fashion that makes as few physical assumptions as possible.

We use the SFD dust map as a template of the $\pi^{0}$ gamma foreground. The correlation between Fermi gamma-ray maps and the SFD dust map is striking, and most of the ISM emission is removed by this subtraction. To reveal the structure deeper into the plane, a simple disk model is subtracted (Su et al. 2010). ${ }^{12}$ The disk model mostly removes the IC gamma rays produced by CR electrons interacting with the ISRF including CMB, infrared, and optical photons; such electrons are believed to be mostly injected in the Galactic disk by supernova shock acceleration

\footnotetext{
10 http://fermi.gsfc.nasa.gov/ssc/data/

11 Available from fermi.gsfc.nasa.gov/ssc/data/.

12 The functional form of this disk template is $(\csc |b|)-1$ in latitude and a Gaussian $\left(\sigma_{\ell}=30^{\circ}\right)$ in longitude.
} 
Table 1

The Template-fitting Coefficients and Errors Correspond to Figures 5 and 6

\begin{tabular}{|c|c|c|c|c|c|c|c|}
\hline $\begin{array}{l}E \text { Range } \\
(\mathrm{GeV})\end{array}$ & Energy & Uniform & SFD Dust & Simple Disk & Bubble & $\begin{array}{l}\text { Bubble } \\
(1.6 \mathrm{yr})\end{array}$ & $\overline{\text { South Cocoon }}$ \\
\hline $0.3-0.5$ & 0.4 & $1.376 \pm 0.007$ & $1.602 \pm 0.019$ & $0.451 \pm 0.011$ & $0.024 \pm 0.020$ & $0.035 \pm 0.033$ & $0.373 \pm 0.055$ \\
\hline $0.5-0.9$ & 0.7 & $1.175 \pm 0.007$ & $1.696 \pm 0.019$ & $0.458 \pm 0.012$ & $0.128 \pm 0.021$ & $0.211 \pm 0.037$ & $0.315 \pm 0.060$ \\
\hline $0.9-1.7$ & 1.3 & $0.897 \pm 0.007$ & $1.489 \pm 0.019$ & $0.383 \pm 0.012$ & $0.167 \pm 0.022$ & $0.321 \pm 0.044$ & $0.415 \pm 0.066$ \\
\hline $1.7-3.0$ & 2.2 & $0.734 \pm 0.006$ & $1.104 \pm 0.016$ & $0.324 \pm 0.010$ & $0.290 \pm 0.018$ & $0.436 \pm 0.036$ & $0.264 \pm 0.054$ \\
\hline $3.0-5.3$ & 4.0 & $0.562 \pm 0.007$ & $0.778 \pm 0.017$ & $0.249 \pm 0.011$ & $0.295 \pm 0.021$ & $0.353 \pm 0.043$ & $0.321 \pm 0.064$ \\
\hline $5.3-9.5$ & 7.1 & $0.500 \pm 0.008$ & $0.475 \pm 0.020$ & $0.170 \pm 0.013$ & $0.363 \pm 0.025$ & $0.343 \pm 0.049$ & $0.306 \pm 0.078$ \\
\hline $9.5-16.9$ & 12.7 & $0.392 \pm 0.009$ & $0.305 \pm 0.023$ & $0.117 \pm 0.015$ & $0.365 \pm 0.030$ & $0.205 \pm 0.055$ & $0.298 \pm 0.094$ \\
\hline $16.9-30.0$ & 22.5 & $0.287 \pm 0.011$ & $0.236 \pm 0.026$ & $0.111 \pm 0.018$ & $0.307 \pm 0.035$ & $0.263 \pm 0.068$ & $0.473 \pm 0.119$ \\
\hline $30.0-53.3$ & 40.0 & $0.244 \pm 0.013$ & $0.156 \pm 0.030$ & $0.028 \pm 0.020$ & $0.279 \pm 0.041$ & $0.217 \pm 0.083$ & $0.051 \pm 0.125$ \\
\hline $53.3-94.9$ & 71.1 & $0.169 \pm 0.014$ & $0.170 \pm 0.036$ & $0.014 \pm 0.022$ & $0.309 \pm 0.050$ & $0.251 \pm 0.120$ & $0.132 \pm 0.152$ \\
\hline $94.9-168.7$ & 126.5 & $0.130 \pm 0.016$ & $0.060 \pm 0.039$ & $0.014 \pm 0.025$ & $0.241 \pm 0.057$ & $0.319 \pm 0.162$ & $0.079 \pm 0.180$ \\
\hline $168.7-300.0$ & 225.0 & $0.086 \pm 0.017$ & $0.045 \pm 0.040$ & $-0.010 \pm 0.027$ & $0.040 \pm 0.052$ & $-0.015 \pm 0.194$ & $0.071 \pm 0.173$ \\
\hline
\end{tabular}

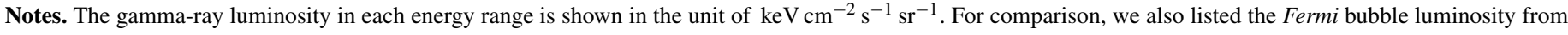
Su et al. (2010).

Table 2

The Template-fitting Coefficients and Errors of the North and South Jets Correspond to Figures 5 and 6

\begin{tabular}{lrrc}
\hline \hline $\begin{array}{l}E \text { Range } \\
(\mathrm{GeV})\end{array}$ & Energy & North Jet & South Jet \\
\hline $0.3-0.9$ & 0.6 & $0.235 \pm 0.139$ & $0.107 \pm 0.112$ \\
$0.9-3.0$ & 1.8 & $0.248 \pm 0.163$ & $0.208 \pm 0.115$ \\
$3.0-9.5$ & 5.6 & $0.248 \pm 0.170$ & $0.002 \pm 0.120$ \\
$9.5-30.0$ & 17.6 & $-0.036 \pm 0.223$ & $0.318 \pm 0.182$ \\
$30.0-94.9$ & 55.6 & $0.526 \pm 0.339$ & $0.656 \pm 0.266$ \\
$94.9-300.0$ & 175.7 & $0.151 \pm 0.446$ & $0.770 \pm 0.395$ \\
\hline
\end{tabular}

Note. The gamma-ray luminosity in each energy range is shown in the unit of $\mathrm{keV} \mathrm{cm}^{-2} \mathrm{~s}^{-1} \mathrm{sr}^{-1}$.

before diffusing outward. The two jets are symmetric with respect to the Galactic plane and well centered on the GC, thus they are unlikely to be local structures.

\section{GAMMA-RAY COCOON AND EVIDENCE OF GALACTIC JET}

The gamma-ray cocoon in the south is visible in the threeyear LAT maps at energies from 1 to $50 \mathrm{GeV}$ (Figure 1). By inspection, the major symmetric axis of the cocoon lines up with the $\mathrm{GC}$ to within $\sim 5^{\circ}$, and the major to minor axis ratio is about a factor of three. The gamma-ray luminosity of the south cocoon with Galactic latitude $|b|>20^{\circ}$ and in the energy range $1-100 \mathrm{GeV}$ is $(5.5 \pm 0.45) \times 10^{35} \mathrm{erg} \mathrm{s}^{-1}$. The morphology of the gamma-ray cocoon resembles the observed radio cocoon structures of Fanaroff-Riley type II (Fanaroff \& Riley 1974) active radio galaxies (e.g., Cygnus A), which have been found surrounding collimated large-scale jets and may be formed by backflow of magnetized jet plasma. How the gamma-ray cocoon system formed and retains its tight columnar shape despite traveling from the GC for $\sim 10 \mathrm{kpc}$ is intriguing. The presence of this large-scale cocoon suggests collimated injection of highenergy particles from the inner Galaxy.

In Figure 2, we show the 0.8-3.2 GeV gamma-ray map of the inner Galaxy. A pair of collimated linear features is revealed in Figure 2, with similar morphology in each energy bin. There are no other apparent large-scale features in the residual maps. The gamma-ray jets do not appear to be associated with the well-known Loop I structure (Berkhuijsen 1973).
The north jet extends from the GC to Galactic coordinates $(\ell, b)=(-11,40)$, and the south jet extends from the GC down to $(\ell, b)=(11,-44)$. Although the jets are faint, three lines of evidence suggest that they are real: (1) the jets both emanate from the GC, in nearly opposite directions; (2) the jets extend away from the GC to about the edge of the previously discovered Fermi bubble structure (Su et al. 2010); and (3) the south jet aligns with the symmetry axis of the cocoon structure. The morphology of the jet and cocoon gamma-ray feature and the possible association with the Fermi bubbles strongly suggests the GC origin of these structures and implies recent activity toward the inner Galaxy.

On a much smaller scale, Chandra X-ray observations have revealed a faint jet-like feature at the subparsec scale, pointing toward Sgr A*(Muno et al. 2008). It is tempting to interpret this feature as a jet of synchrotron-emitting particles ejected from the SMBH. However, due to its much smaller size and $\sim 15^{\circ}$ misalignment with the gamma-ray jet, there is no clear association. Limits on the gamma-ray jet from other wavelengths do not strongly constrain its nature, underscoring the need for further multi-wavelength studies.

The projected direction of the gamma-ray jets is about $15^{\circ}$ from the north-south axis of the Galaxy. If the gamma-ray jets constitute the projection of double large-scale jets symmetric to the Galactic plane and the GC, taking the distance from the solar system to the GC $R_{\odot}=8.5 \mathrm{kpc}$, then each projected jet is $\sim 10 \mathrm{kpc}$. The small angle of the projected jet relative to the north-south Galactic axis suggests that the apparent length of $\sim 10 \mathrm{kpc}$ is a good approximation of the spatial scale of the Galactic jet. The width of the jets appears to be $\lesssim 5^{\circ}$. The total luminosity of the north and south jet-like features is $(1.8 \pm 0.35) \times 10^{35} \mathrm{erg} \mathrm{s}^{-1}$ at $1-100 \mathrm{GeV}$ (see Table 2). The jets do not align with any plausible artifacts relating to the Fermi orbit or scan direction, or any other known systematics. The north and south jets have similar integrated gamma-ray flux, so there is no evidence that the jets are close to the line of sight; otherwise, the approaching jet would appear substantially Doppler brightened relative to the receding one.

This gamma-ray jet/cocoon structure is not visible in the ROSAT All-Sky Survey of soft X-ray (Snowden et al. 1997), Wilkinson Microwave Anisotropy Probe (WMAP) microwave maps, radio maps at $408 \mathrm{MHz}$ (Haslam et al. 1982a), or any other available radio maps. We estimate expected radio 

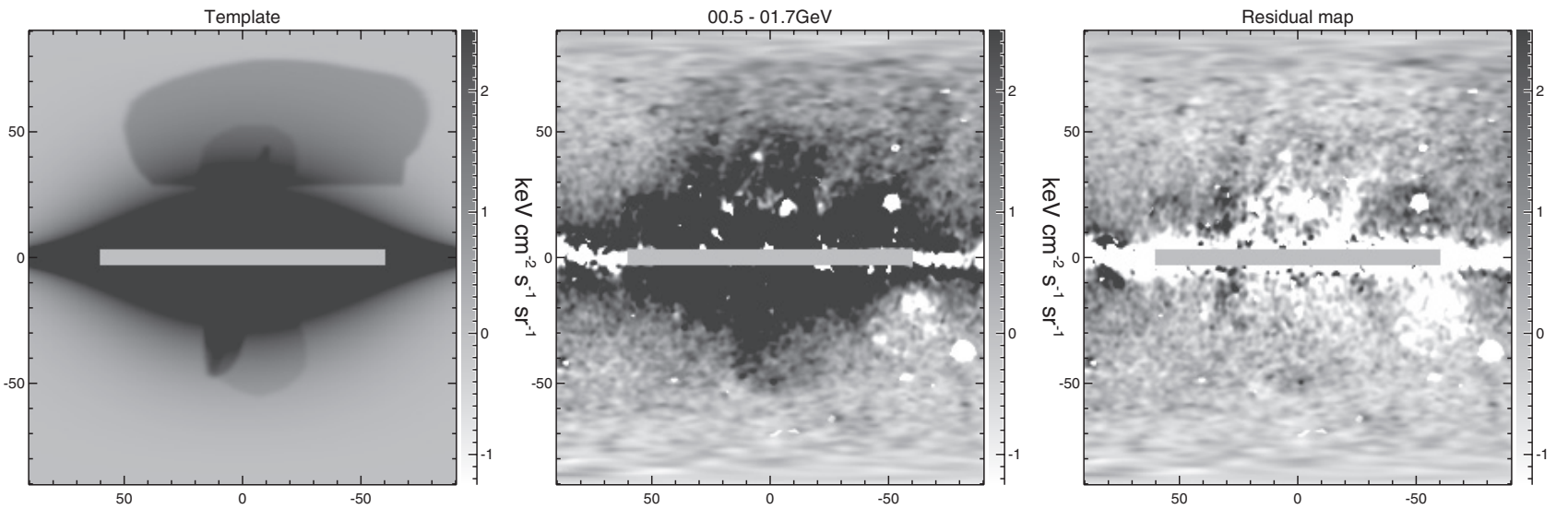

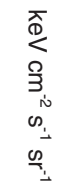
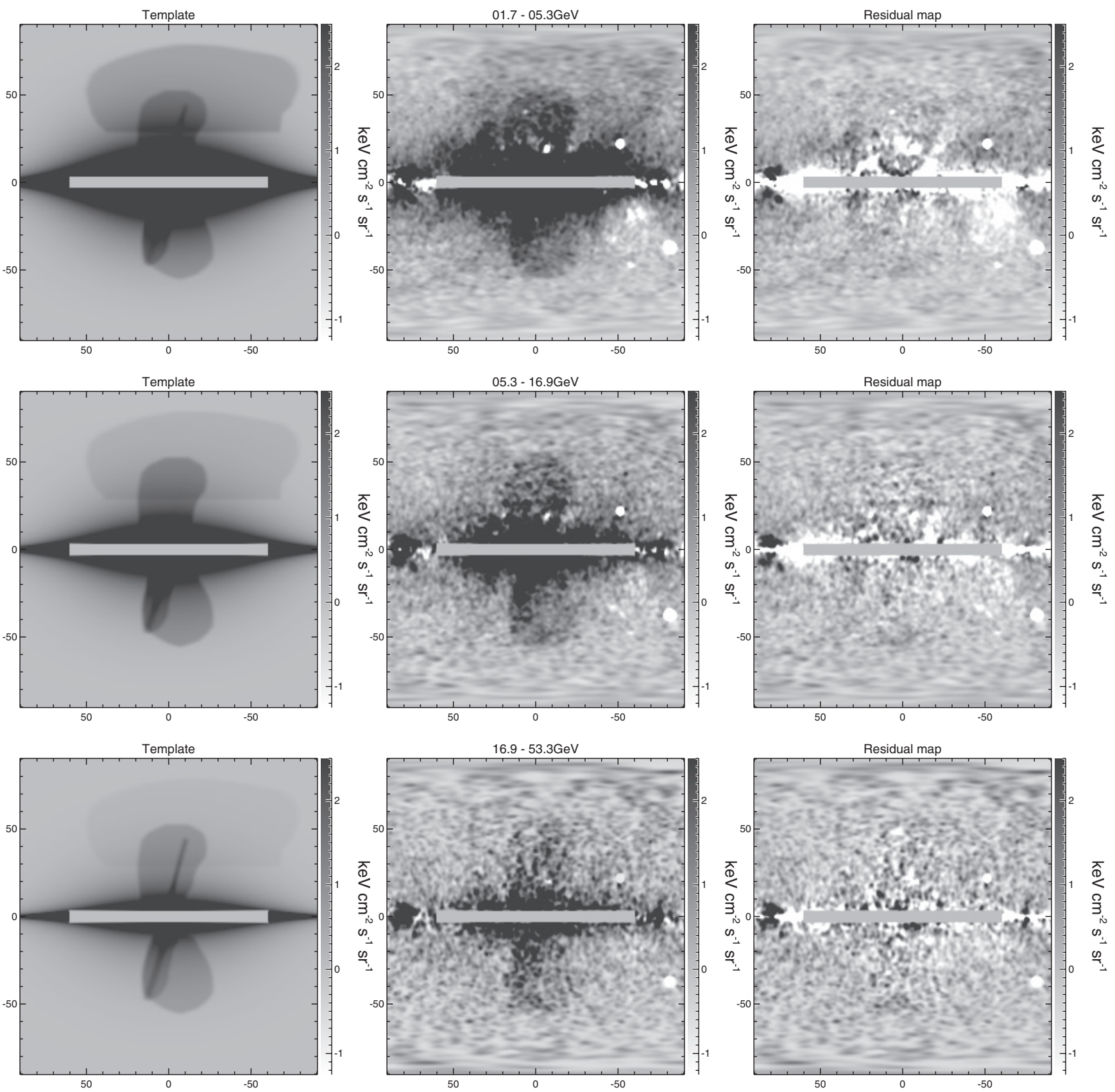

Figure 6. Same as Figure 4, except we include the north jet template. 


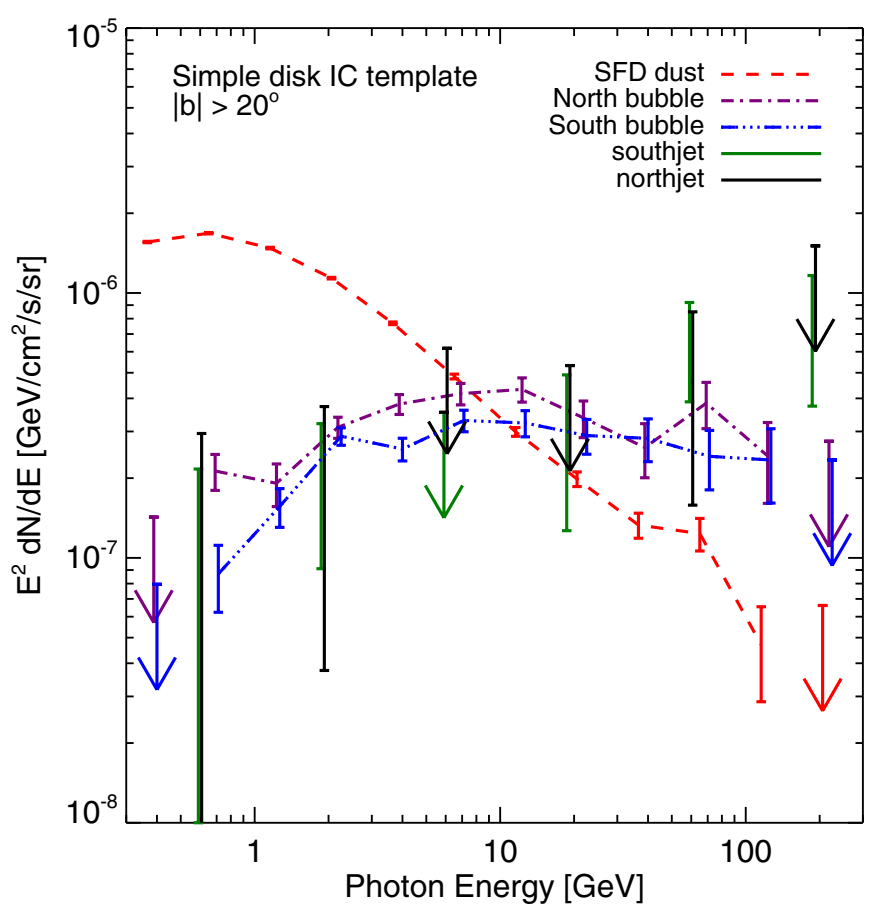

Figure 7. Same as Figure 1, except we add the north jet template and split the Fermi bubble into north and south bubbles separately. The south cocoon template is included in the fit. The north and south jet spectra are consistently hard. The fitting results are not significantly affected by the bubble template splitting.

(A color version of this figure is available in the online journal.)

spectra in Section 6 corresponding to electron spectral indices $1.5<\gamma<2.5$, and find that over this range, current full-sky radio surveys are not sensitive enough to detect the jet. However, combining the gamma-ray energy spectrum along with the radio limits, we find that the CR electrons producing the jet have a hard spectrum with a spectral index of $\gtrsim-2$.

We now turn to the relation between the jet/cocoon and the Fermi bubbles. We recently discovered two giant gammaray bubbles with a total luminosity from 1 to $100 \mathrm{GeV}$ of $2.0 \times 10^{37} \mathrm{erg} \mathrm{s}^{-1},{ }^{13}$ extending $\sim 50^{\circ}$ above and below the $\mathrm{GC}$, with a width of $\sim 40^{\circ}$ in longitude, and found them to be spatially correlated with a hard-spectrum microwave excess (known as the WMAP haze; Finkbeiner 2004) and large-scale $\mathrm{X}$-ray features (Su et al. 2010). Galactic shock waves produced by energetic explosions at the Galactic nucleus or by a high rate of supernova explosions in the nuclear disk will be channeled by the decreasing ambient gas density in directions perpendicular to the Galactic plane.

The gamma-ray emission associated with these bubbles has a significantly harder spectrum $\left(d N / d E \sim E^{-2}\right)$ than the IC emission from electrons in the Galactic disk, or the gamma rays produced by decay of $\pi^{0}$ from proton-ISM collisions. The Fermi bubbles are likely formed during an active phase in the GC $\sim 10^{6}-10^{7}$ years ago with jets ejected from the central SMBH. The bubble region might consist of decelerated jet material, radiating isotropically. Observational data and numerical simulations indicate that the energy required to form the bubbles is of the order of $10^{55}-10^{58} \mathrm{erg}$ (Su et al. 2010; Guo \& Mathews 2011; Guo et al. 2011). Even though the current jet luminosity is relatively faint, the discovery of the jet/cocoon

\footnotetext{
13 We note that in Su et al. (2010), the total gamma-ray luminosity of the Fermi bubbles was misquoted as $4.0 \times 10^{37} \mathrm{erg} \mathrm{s}^{-1}$.
}

system generally supports the AGN hypothesis for the origin of the bubbles.

\section{ENERGY SPECTRUM OF THE GAMMA-RAY COCOON AND JETS}

We have shown in Figure 1 that the gamma-ray jets, the cocoon, and the Fermi bubbles all have a harder spectrum than other large-scale diffuse ISM emission. In order to measure the hardness of the spectrum and to explore possible mechanisms responsible for the observed gamma-ray emission, we maximize the likelihood that the maps are described by a linear combination of spatial templates, one for each gamma-ray emission mechanism. We fit a coefficient to each emission component using a multi-linear regression of simple templates, one energy bin at a time. By combining results from 12 logarithmically spaced energy bands from $300 \mathrm{MeV}$ to $300 \mathrm{GeV}$, we determine a spectral energy distribution (SED) for each component. In each fit, we maximize the Poisson likelihood of a simple diffuse emission model involving the seven following templates: the SFD map of Galactic dust (Schlegel et al. 1998), the simple disk model, the Fermi bubbles, a template of the Loop I structure ( $\mathrm{Su}$ et al. 2010), the gamma-ray cocoon, the gamma-ray south jet, and a uniform background as template to account for background gamma-ray emission and CR contamination (Su et al. 2010). All maps and templates have been smoothed by a Gaussian kernel with FWHM of $2^{\circ}$ for the regression analysis. Systematic uncertainties are dominated by the imperfect representation of the diffuse emission by these simple templates. We feel that this analysis is sufficient for a rough characterization of the cocoon and jet spectra, and provides motivation for more thorough analysis using a more physical model in the future.

For each set of model parameters, we compute the Poisson log likelihood,

$$
\ln \mathcal{L}=\sum_{i} k_{i} \ln \mu_{i}-\mu_{i}-\ln \left(k_{i} !\right),
$$

where $\mu_{i}$ is the synthetic counts map (i.e., linear combination of templates times exposure map) at pixel $i$ and $k$ is the map of observed counts. The last term is a function only of the observed maps. We compute errors in the Gaussian approximation by inverting the matrix of second partial derivatives of $-\ln \mathcal{L}$ to obtain the covariance matrix, and taking the square root of the diagonals. The $1 \sigma$ Gaussian error corresponds to $\Delta \ln \mathcal{L}=1 / 2$. We refer to Appendix B of Dobler et al. (2010) for more details of the likelihood analysis. Maps of the models are constructed from linear combinations of these templates, and the corresponding energy spectrum and residual (data minus model) maps in various energy bins are shown in Figures 3 and 4, respectively. In this fit, we mask out all pixels with Galactic latitude $|b|<20^{\circ}$.

So far, we have described the procedure for obtaining the correlation coefficient for each template at each energy. However, some templates have units (e.g., the SFD dust map is in magnitudes of $E_{B-V}$ reddening), so the correlation coefficient has unusual units (e.g., gamma-ray emission per magnitude). In such a case, we multiply the correlation spectrum by the average SFD value in the $\left(|b|>20^{\circ}\right)$ bubble region, to yield the average spectrum of dust-correlated gamma-ray emission in this region. For the uniform, Loop I, cocoon, jet, and bubble templates, no renormalization is done. These templates are simply ones and zeros (smoothed to the appropriate PSF), so the south jet spectrum is simply the spectrum of the south jet template shown in, 

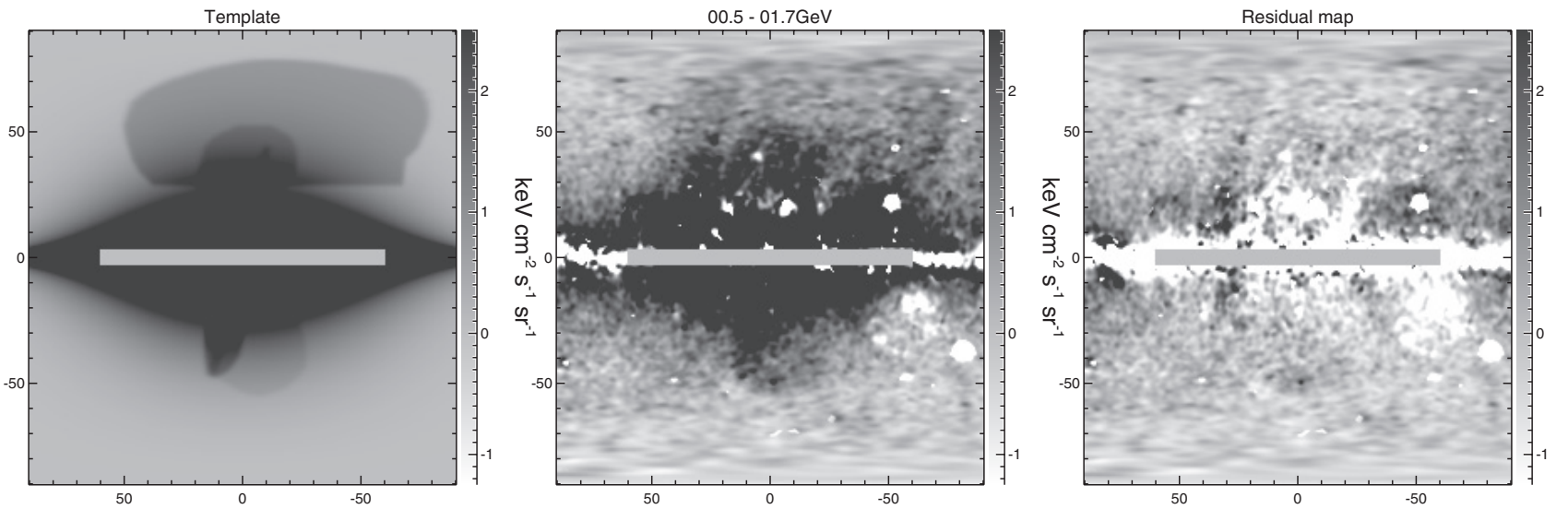

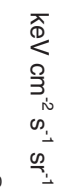
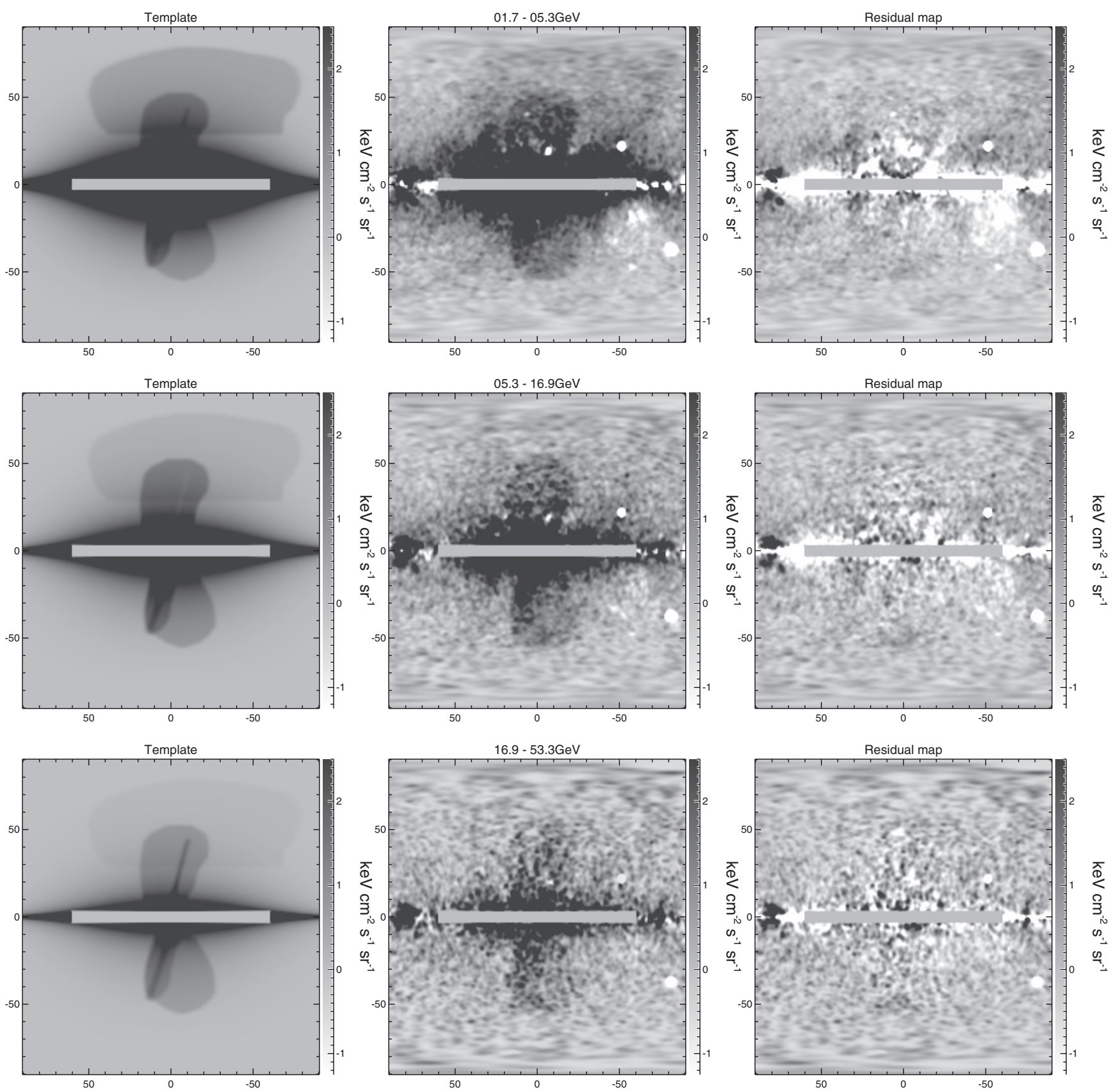

Figure 8. Same as Figure 1, except we add the north jet template and split the bubble template to north and south separately. Note that the south cocoon template is included in the fitting. The north and south jet spectra are consistently hard. 
e.g., the left panels of Figure 4, not the mean of this template over the whole bubble region.

The energy spectra for $\pi^{0}$ emission, bremsstrahlung, and IC scattering can be calculated using a sample GALPROP model ${ }^{14}$ (tuned to match locally measured protons and anti-protons as well as locally measured electrons at $\sim 20-30 \mathrm{GeV}$ ), as an indication of the expected spectral shapes. We have shown in Su et al. (2010) that the energy spectra for the SFD and the simple disk template reasonably match the model expectations. The dust map mostly traces the $\pi^{0}$ emission, and the simple disk model resembles a combination of IC and bremsstrahlung emission. The spectrum for emission correlated with the gammaray jet and cocoon is clearly significantly harder than either of these components, consistent with a flat spectrum in $E^{2} d N / d E$. This fact, coupled with the distinct spatial morphology of the jet/cocoon system, indicates that if these gamma-ray features are generated by IC scattering of ISRFs, then a separate electron population must exist in the jet/cocoon. We also note that the spectrum of the jet/cocoon template does not fall off significantly at energy $\lesssim 1 \mathrm{GeV}$ as the bubble spectrum does. The fitting coefficients and corresponding errors of each template are listed in Tables 1 and 2.

The hardness of this spectrum may be the key to deciphering the origin of the gamma-ray jet/cocoon. In Su et al. (2010), we show that at lower energy $(E \lesssim 1 \mathrm{GeV})$ the bubble spectrum falls sharply with decreasing energy (becomes dramatically harder than -2). With reduced statistical error from three-year LAT data and the new Pass 7 ULTRACLEAN event selection, we confirm significant falling of the bubble spectrum at lower energy but not the jet and cocoon components. This is true whether we perform the entire analysis with front-converting events only, or use front converting at $E<1 \mathrm{GeV}$ and both front and back converting at $E>1 \mathrm{GeV}$ as usual.

The null hypothesis of zero intensity of the north (south) jet is ruled out by $3.1 \sigma(4.1 \sigma)$, respectively, and $5.2 \sigma$ jointly for the whole jet structure. The same fit simultaneously finds the cocoon with $12 \sigma$ significance. Since the region south of the GC has less foreground emission than the north (where $\rho$ Oph is), we focus on the south jet/cocoon for our analysis. Figure 3 (right panel) shows the resulting energy spectrum of the gamma rays associated with the cocoon and the south jet. Like the Fermi bubbles, the energy spectrum of the jet/cocoon is harder than other diffuse gamma-ray components, although the cocoon has a spectrum at $<1 \mathrm{GeV}$ different from the Fermi bubbles, which suggests its origin from a distinct population of electron CRs. The cocoon spectrum is consistent with $E^{2} d N / d E \sim$ constant, and the north and south jet structures have an energy spectrum of $E^{2} d N / d E \sim E^{0.2 \pm 0.2}$ at latitudes of $|b|>20^{\circ}$. The correlation coefficients for the SFD map and simple disk model are multiplied by the average value of these maps in the bubble region with a $|b|>20^{\circ}$ cut to obtain the associated gamma-ray emission. Given how hard the cocoon/jet spectra are up to $\sim 100 \mathrm{GeV}$, bremsstrahlung can be ruled out as the emission mechanism based on the arguments in Kino et al. (2009).

The gamma-ray jets appear to possess north-south symmetry in morphology without noticeable difference in intensity. To investigate whether there is any spectral difference between the

\footnotetext{
14 GALPROP is a cosmic-ray propagation code. It calculates the steady state solution to the diffusion-energy-loss equation, given the 3D gas distribution, interstellar radiation field, B-field model, CR diffusion assumptions, and many other input parameters (Strong \& Moskalenko 1999). See http://galprop. stanford.edu.
}
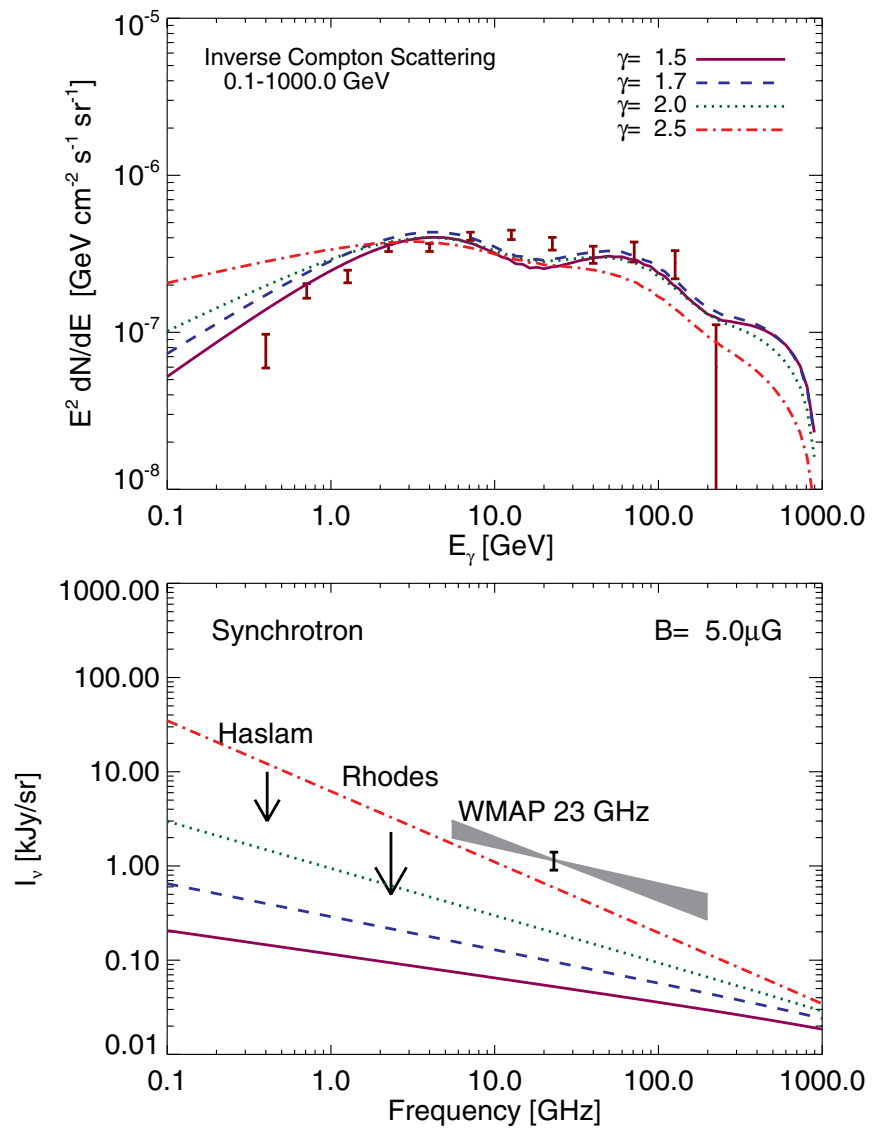

Figure 9. Estimated spectrum of inverse Compton gamma rays (upper panel) and corresponding synchrotron radiation (lower panel) originating from a hard electron spectrum along a line of sight $4 \mathrm{kpc}$ below the Galactic plane (i.e., $\left.b \approx-25^{\circ}\right)$. The steady state electron spectrum is taken to be a power law, $d N / d E \propto E^{-\gamma}$, with index $\gamma=1.5$ (solid black), 1.7 (blue dashed), 2.0 (green dotted), and 2.5 (red dash-dotted) in both the upper and lower panels. In all cases, the CR electron has a range of $[0.1,1000] \mathrm{GeV}$. The interstellar radiation field model is taken from GALPROP version 50p, and the magnetic field is assumed to be $5 \mu \mathrm{G}$ for synchrotron calculation. The data points in the upper panels show the south cocoon emission the same as in Figure 3. The arrows show $3 \sigma$ upper limits rather than data points with $1 \sigma$ error bars, due to the large uncertainties in those energies. The data point in the lower panel shows the magnitude of the $W M A P$ haze averaged over $b=-20^{\circ}$ to $-30^{\circ}$ in the $23 \mathrm{GHz} K$ band, and the gray area indicates the range of synchrotron spectral indices allowed for the WMAP haze (Dobler \& Finkbeiner 2008). Two arrows at lower frequency show the $3 \sigma$ upper limits for the cocoon radio emission from Rhodes/HartRAO $2.326 \mathrm{GHz}$ radio continuum survey (Jonas et al. 1998) and Haslam $0.408 \mathrm{GHz}$ map (Haslam et al. 1982b). The same population of CR electrons can produce both the observed radio/microwave signal and the observed gamma-rays.

(A color version of this figure is available in the online journal.)

north and south jets, we augment the seven-template fit (dust, simple disk, Fermi bubble, cocoon, south jet, uniform, Loop I) with a north jet template. We show the resulting spectrum in Figure 5 and the maps in Figure 6. We then repeat the previous fitting procedure involving the simple disk IC template, but splitting the bubble template into north and south bubble templates, and allow an independent fit of the two jet templates along with the two bubbles. The goal is to identify variations in the intensity and spectral index between the northern and southern jets and the dependence of the jet spectrum on the combination of different emission templates. Even given this freedom, no significant spectral differences are found between the north and south jets or the north and south bubbles. There was no significant improvement of the likelihood for such splitting. The spectrum and the maps are shown in Figures 7 and 8, 

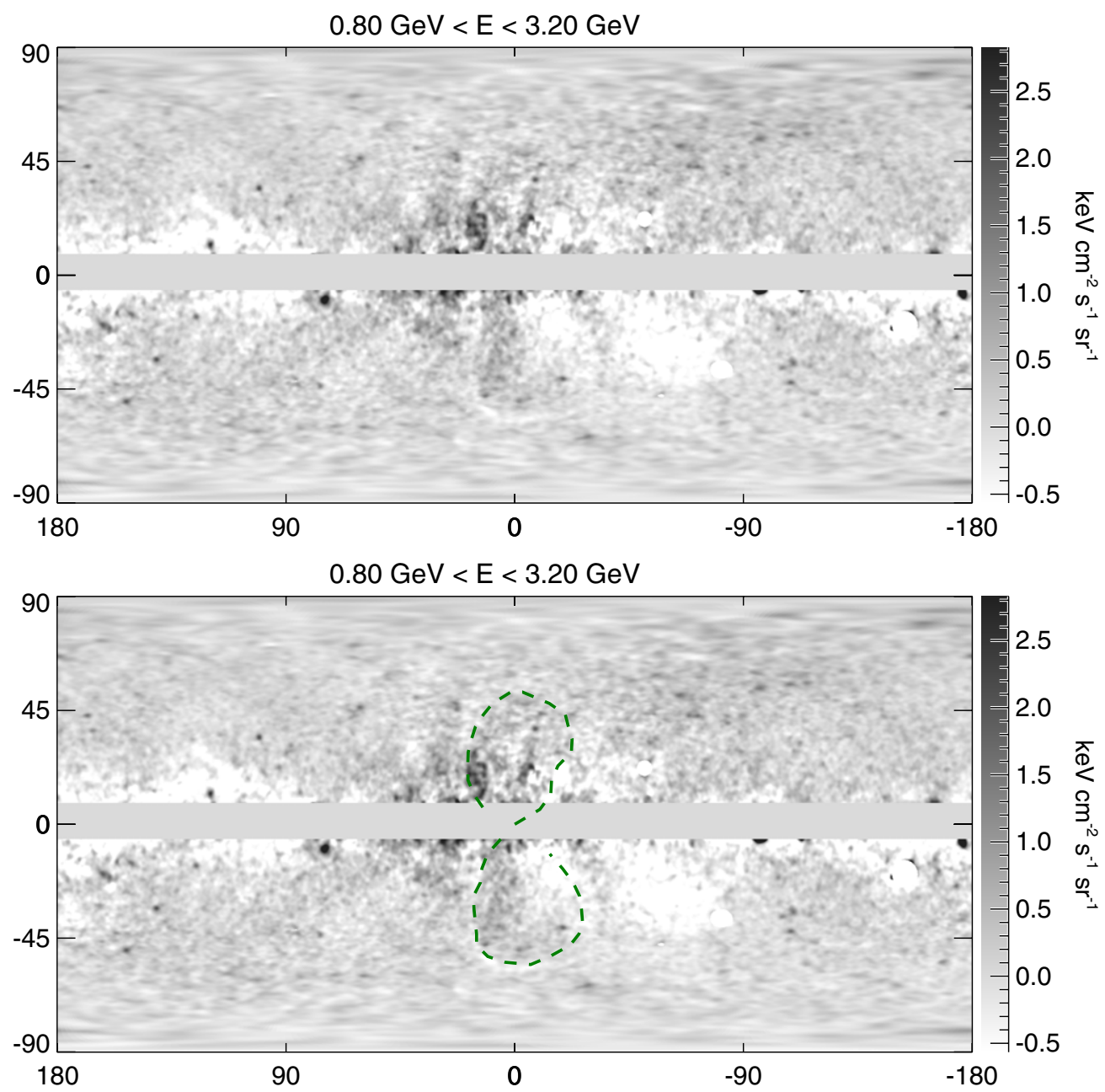

Figure 10. Full-sky residual maps after subtracting the Fermi diffuse emission model from the Fermi-LAT three-year gamma-ray maps. Upper panel: point sources have been subtracted, and large sources, including the inner Galactic disk, have been masked. The Fermi bubble structure has been included in the diffuse emission model. We find no large-scale jet features other than the central Galactic jet toward the inner Galaxy. The Galactic jet feature is crossing the Galactic center, and aligns with the long axis of the cocoon structure. Lower panel: the Fermi bubble edge has been marked in green dashed circles above and below the Galactic center, overplotted on the gamma-ray map the same as the upper panel. Both the north and the south jets, approximately end on the edge of the Fermi bubble structure.

(A color version of this figure is available in the online journal.)

respectively. Our conclusion is that the gamma-ray jets appear to be north-south symmetric, both with a hard spectrum. This statement is largely independent of our choice of template for the disk IC emission. In summary, we found no apparent difference between the north and the south jets, both in morphology and in energy spectrum, which indicates they might share the same origin.

\section{EXPECTED RADIO LUMINOSITY OF THE JET}

In order to estimate the expected radio luminosity of the jet, we must either have a physical model of the radio and gamma-ray emission mechanisms, or a prototype object for comparison. Very Long Baseline Array $5 \mathrm{GHz}$ observations of blazars detected by LAT provide one benchmark (Linford et al. 2011). This sample of blazars shows a median $5 \mathrm{GHz}$ flux of $\sim 300 \mathrm{mJy}$ and a median gamma-ray flux $(0.1-100 \mathrm{GeV})$ of $3 \times$ $10^{-8}$ photons $\mathrm{cm}^{-2} \mathrm{~s}^{-1}$, although individual objects can deviate from this ratio by an order of magnitude or more. BL Lac objects have a spectrum of $d N / d E \sim E^{-1.8}$ in this energy range, and therefore have $1 / 15$ of their $0.1 \mathrm{GeV}<E<100 \mathrm{GeV}$ photons in the range $1-2 \mathrm{GeV}$. Assuming such a spectrum, a $5 \mathrm{GHz}$ flux of $300 \mathrm{mJy}$ corresponds to $4 \times 10^{-9} \mathrm{GeV} \mathrm{cm}^{-2} \mathrm{~s}^{-1}(1-2 \mathrm{GeV})$. Therefore, the $5 \mathrm{GHz}$ radio signal corresponding to our observed $4 \times 10^{-7} \mathrm{GeV} \mathrm{cm}^{-2} \mathrm{~s}^{-1} \mathrm{sr}^{-1}$ would be $30 \mathrm{Jy} \mathrm{sr}^{-1}$, much smaller than our radio limits in Figure 9. However, because BL Lac objects are thought to contain a relativistic jet pointed along the line of sight, they may be a poor analog for the Galactic jet.

An alternative is to take a magnetic field constrained by radio and Faraday rotation measure observations (Sun et al. 2008) and an ISRF (taken from GALPROP, $4 \mathrm{kpc}$ below the GC) to compute the IC gammas and synchrotron given a power-law CR electron spectrum. The high-energy cutoff of the electron spectrum is important for the IC gammas, especially for spectra harder than $d N / d E \sim E^{-2}$. As an example, we have computed the IC gamma-ray and synchrotron emission for four power laws, with spectral index in the range $\gamma=1.5-2.5$ with a cutoff at $E_{\text {electron }}=1 \mathrm{TeV}$ (Figure 9). The electron spectra are normalized such that the IC gammas go through the data points. The synchrotron is then computed assuming a $5 \mu \mathrm{G}$ magnetic field and the GALPROP ISRF (Porter et al. 2008) at $4 \mathrm{kpc}$ below the Galactic plane. Upper limits from maps at $408 \mathrm{MHz}$ (Haslam 


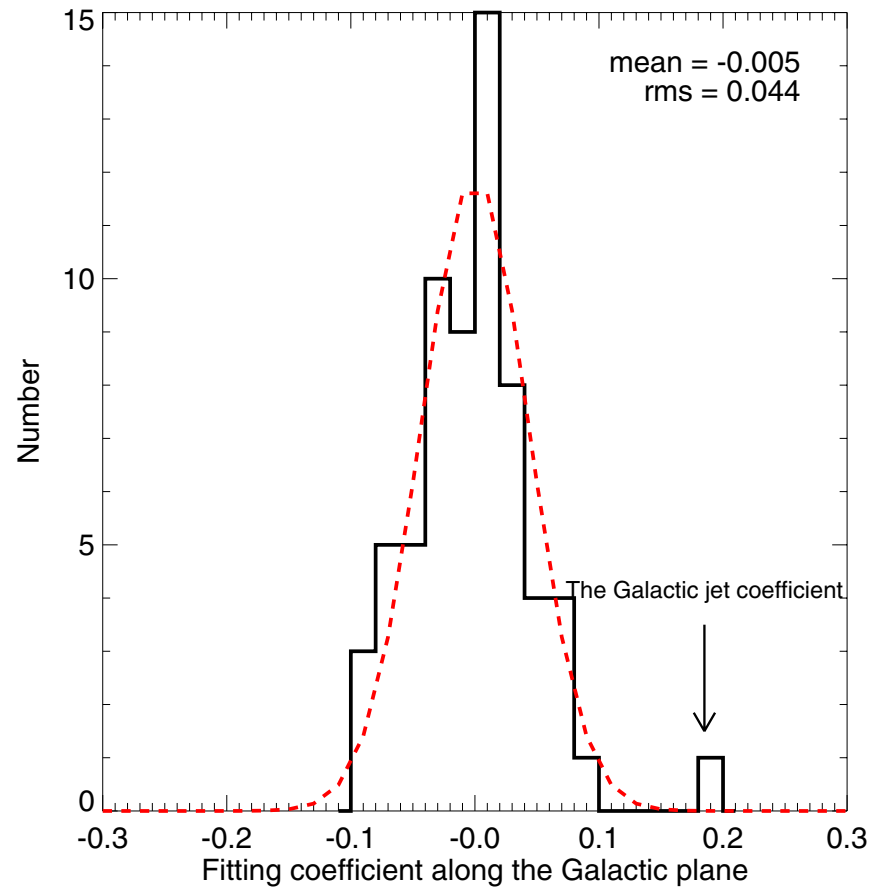

Figure 11. As a verification of our uncertainty estimate for the Galactic jet structure, we have rotated the south jet and cocoon templates in longitude around the sky, and determined the best-fit coefficient for each case. This histogram shows the distribution of the coefficients overplotted with the fitted Gaussian distribution. The coefficient distribution is well centered around zero and well fitted with a Gaussian noise distribution. The south jet template with a coefficient 0.182 is about $4 \sigma$ significance from the background noise estimation. The estimated uncertainty of the jet template agrees well with our estimation of the significance of the south jet based on the Poisson likelihood fitting.

(A color version of this figure is available in the online journal.)

et al. 1982b) and $2.3 \mathrm{GHz}$ (Jonas et al. 1998), and a data point from WMAP $23 \mathrm{GHz} 4 \mathrm{kpc}$ off the disk (which together serve as an upper limit for the jet brightness; Dobler \& Finkbeiner 2008) are included. Most of the lines pass below the limits, making it plausible that the radio signal corresponding to a gamma-ray jet would not yet have been observed. Additional uncertainty arises from the fact that the $1 \mathrm{GHz}$ radio signal is produced by order $1 \mathrm{GeV}$ electrons, whereas the gamma-ray signal arises from order 100-1000 GeV electrons. If the electron spectrum has any downward curvature over this range in energy, then the radio/microwave limits become even less constraining. Another uncertainty comes from the choice of energy cutoff for the power law. We considered spectra with cutoffs at 3 and $5 \mathrm{TeV}$, with index $\gamma \sim 3$ to fit the gamma-ray data. Such spectra predict a synchrotron signal bright enough to be ruled out by the radio data. This implies that the required CR spectrum is much harder than the locally measured index of $\gamma \sim 3-3.25$.

\section{STATISTICAL SIGNIFICANCE OF THE JET}

In Figure 10, we show the full-sky residual maps at 0.8-3.2 GeV after subtracting the Fermi diffuse emission model. This figure is the full-sky version of Figure 1. We identify a pair of Galactic jet features toward the inner Galaxy, present both above and below the Galactic plane and aligned with the GC. There are no other significant large-scale jet structures appearing in the full-sky gamma-ray map.

Our best-fit values are derived from a maximum likelihood analysis, in which we maximize the Poisson probability of observing the observed counts, given a set of model parameters. The error bars are derived from the parameter covariance matrix. In our previous work (Su et al. 2010), we analyzed numerous mock maps to verify that our code gives unbiased results with the correct uncertainties, at least in the case where the model (plus Poisson noise) is a good description of the data. However, our fits contain systematic residuals, and it is necessary to investigate to what extent these might be able to mimic a signal. To this end, we have repeated the seven-component fit with the cocoon and jet structures shifted around the sky at $5 \mathrm{deg}$ intervals in longitude $\ell$, and determined the best-fit coefficient for each case by fitting the same templates as in Figure 3. By placing the templates at different positions along the longitudes where we expect no significant large-scale diffuse structures, we can measure the rms of the jet and cocoon coefficients, and this rms may exceed that due to the Poisson noise. In some parts of the sky (e.g., $\ell \sim 290^{\circ}-320^{\circ}$ ), the $\pi^{0}$ emission is somewhat oversubtracted, which pushes the cocoon coefficient negative, effectively renormalizing the sky background for the jet. As expected, the distribution of jet template coefficients (shown in Figure 11) is centered around zero, consistent with a Gaussian of mean zero and $\sigma=0.044$. The Galactic jet coefficient is more than $4 \sigma$ out of the background noise distribution, which is consistent with the estimated significance of the south jet derived from the covariance matrix of our Poisson likelihood fit. We interpret this agreement to mean that systematic errors in foreground modeling have not substantially distorted the meaning of the south jet's $4 \sigma$ formal significance.

\section{DISCUSSION AND CONCLUSION}

Given the alignment of the cocoon with the southern bubble edge, the cocoon/jet is probably associated with the bubble, and their origins may be intertwined. A possible scenario in which a jet creates both the cocoon and the Fermi bubble is as follows. The propagation of a large-scale relativistic jet may generate a double bow-shock structure at the head of the jet (Blandford \& Rees 1974; Scheuer 1974). Entrained energy and matter are pushed aside due to a high-pressure gradient and create a hot cocoon around the jet. The cocoon applies sufficiently high pressure to collimate the jet and substantially reduce its opening angle. Continuous injection of relativistic electrons can be produced in the forward, reverse, and re-collimation shocks emitting synchrotron radiation and gamma-ray IC emission. The jets deliver kinetic energy to the surrounding interstellar gas and the jet/medium interactions could be strong enough to accelerate particles and produce non-thermal radiation. In this scenario, as the jets emerge from the GC, they expand freely until they are re-collimated when their ram pressure falls to the thermal pressure of the surrounding cocoon. The jet decelerates once the accumulated mass of the swept up ISM gas becomes similar to that carried by the jet. The cocoon and Fermi bubble could be explained by jet-shocked material (reconfinement region and cocoon) and the ambient material could be shocked by the bow shock. However, the $15^{\circ}$ misalignment between the jet/cocoon and the bubbles, and the absence of an obvious northern cocoon, suggests that this is not the whole story. We cannot rule out the possibility that the Fermi bubbles were formed by an earlier energetic event, while the jet might be produced more recently and be penetrating through the older structure.

The gamma-ray jet is the first collimated jet structure found in gamma rays and the only collimated jet close enough to resolve with Fermi-LAT. The presence of such a large-scale 
Galactic jet in our MW provides an ideal nearby laboratory for studying basic questions about jet formation, acceleration, and collimation. It is likely that similar systems are not uncommon. For example, the recently observed unusual transient source (Swift J164449.3+573451) has been understood as a newly formed relativistic outflow launched by transient accretion onto an SMBH with mass similar to the GC SMBH (Zauderer et al. 2011; Burrows et al. 2011). It provides the evidence that a normal galaxy can transform to an AGN-like phase and produce a relativistic jet. Similar structures on much larger scales have been found in galaxy clusters with radio lobes inflated by the jets of SMBH at the center of powerful radio galaxies (McNamara et al. 2005).

Follow-up observations at other wavelengths are required to advance our understanding of the jet and cocoon structures, and their relation to the Fermi bubbles. In coming years, eRosita will survey the whole sky in X-rays with approximately 30 times ROSAT sensitivity, in the medium energy $\mathrm{X}$-ray range up to $10 \mathrm{keV}$ with an unprecedented spectral and angular resolution (Cappelluti et al. 2011). It has the ability to reveal important information about the state of the material in the jet, cocoon, and bubble structures. Such multi-wavelength exploration will advance our understanding not only of our own Galaxy, but of black hole accretion and jet formation in general.

It is a pleasure to acknowledge helpful conversations with Aneta Siemiginowska, Martin Elvis, Feng Yuan, and Roman Shcherbakov. We acknowledge the use of public data from the Fermi data archive at http://fermi.gsfc.nasa.gov/ssc/. M.S. and D.P.F. are partially supported by the NASA Fermi Guest Investigator Program. This research made use of the NASA Astrophysics Data System (ADS) and the IDL Astronomy User's Library at Goddard (available at http://idlastro.gsfc.nasa.gov).

\section{REFERENCES}

Antonuccio-Delogu, V., \& Silk, J. 2008, MNRAS, 389, 1750

Atwood, W. B., Abdo, A. A., Ackermann, M., et al. 2009, ApJ, 697, 1071

Berkhuijsen, E. M. 1973, A\&A, 24, 143

Blandford, R. D., \& Payne, D. G. 1982, MNRAS, 199, 883

Blandford, R. D., \& Rees, M. J. 1974, MNRAS, 169, 395

Blandford, R. D., \& Znajek, R. L. 1977, MNRAS, 179, 433

Bridle, A. H., \& Perley, R. A. 1984, ARA\&A, 22, 319

Burrows, D. N., Kennea, J. A., Ghisellini, G., et al. 2011, Nature, 476, 421

Cappelluti, N., Predehl, P., Böhringer, H., et al. 2011, Mem. Soc. Astron. Ital. Suppl., 17, 159

Dobler, G., \& Finkbeiner, D. P. 2008, ApJ, 680, 1222

Dobler, G., Finkbeiner, D. P., Cholis, I., Slatyer, T., \& Weiner, N. 2010, ApJ, 717,825

Fanaroff, B. L., \& Riley, J. M. 1974, MNRAS, 167, 31P

Finkbeiner, D. P. 2004, ApJ, 614, 186

Gehrels, N., \& Michelson, P. 1999, Astropart. Phys., 11, 277

Grenier, I. A., Casandjian, J., \& Terrier, R. 2005, Science, 307, 1292

Guo, F., \& Mathews, W. G. 2011, arXiv:1103.0055

Guo, F., Mathews, W. G., Dobler, G., \& Oh, S. P. 2011, ApJ, submitted (arXiv:1110.0834)

Haslam, C. G. T., Salter, C. J., Stoffel, H., \& Wilson, W. E. 1982a, A\&AS, 47, 1

Haslam, C. G. T., Salter, C. J., Stoffel, H., \& Wilson, W. E. 1982b, A\&AS, 47, 1

Jonas, J. L., Baart, E. E., \& Nicolson, G. D. 1998, MNRAS, 297, 977

Kino, M., Ito, H., Kawakatu, N., \& Nagai, H. 2009, MNRAS, 395, L43

Linford, J. D., Taylor, G. B., Romani, R. W., et al. 2011, ApJ, arXiv:1111.4505

McNamara, B. R., Nulsen, P. E. J., Wise, M. W., et al. 2005, Nature, 433, 45

Morris, M., \& Serabyn, E. 1996, ARA\&A, 34, 645

Muno, M. P., Baganoff, F. K., Brandt, W. N., Morris, M. R., \& Starck, J. 2008, ApJ, 673, 251

Porter, T. A., Moskalenko, I. V., Strong, A. W., Orlando, E., \& Bouchet, L. 2008, ApJ, 682, 400

Scheuer, P. A. G. 1974, MNRAS, 166, 513

Schlegel, D. J., Finkbeiner, D. P., \& Davis, M. 1998, ApJ, 500, 525

Snowden, S. L., Egger, R., Freyberg, M. J., et al. 1997, ApJ, 485, 125

Strong, A. W., \& Moskalenko, I. V. 1999, 4, 255

Su, M., Slatyer, T. R., \& Finkbeiner, D. P. 2010, ApJ, 724, 1044

Sun, X. H., Reich, W., Waelkens, A., \& Enßlin, T. A. 2008, A\&A, 477, 573

Sunyaev, R. A., Markevitch, M., \& Pavlinsky, M. 1993, ApJ, 407, 606

The Fermi-LAT Collaboration 2011, arXiv:1108.1435

Zauderer, B. A., Berger, E., Soderberg, A. M., et al. 2011, Nature, 476, 425 